Service social

\title{
Femmes immigrantes : problématiques et intervention féministe
}

\section{Gisèle Legault}

Volume 42, numéro 1, 1993

Culture et intervention

URI : https://id.erudit.org/iderudit/706600ar

DOI : https://doi.org/10.7202/706600ar

Aller au sommaire du numéro

Éditeur(s)

École de service social de l'Université Laval

ISSN

1708-1734 (numérique)

Découvrir la revue

Citer cet article

Legault, G. (1993). Femmes immigrantes : problématiques et intervention féministe. Service social, 42(1), 63-80. https://doi.org/10.7202/706600ar
Résumé de l'article

L'article traite des principaux problèmes sociaux vécus par les femmes immigrantes et des modalités d'une intervention féministe auprès de ces femmes. La problématique sociale révélée par les écrits et une recherche exploratoire qualitative conduite auprès d'intervenantes sociales des services sociaux publics de la région de Montréal en 1990-1991 a trait principalement à la remise en question / renégociation des rôles sociaux, aux problèmes de santé physique et mentale et à quelques autres problèmes sociaux plus larges. Les modalités d'une intervention féministe auprès des femmes immigrantes sont explicitées autour des trois variables que sont l'analyse des problèmes, les objectifs visés et les stratégies utilisées. 


\section{Femmes immigrantes: problématiques et intervention féministe}

Gisèle LEGAULT

Professeur agrégée, École de service social Université de Montréal

Nous faisions état dans cette revue, il y a quelques années (Legault et RojasBenavente, 1988), des résultats d'une recension des écrits portant sur les femmes immigrantes et le travail social. C'était alors le début d'une recherche exploratoire sur le sujet, recherche maintenant terminée et dont la synthèse a paru récemment (Legault et Lafrenière, 1992). Cette recherche a été centrée essentiellement sur les difficultés de pratique de 40 intervenantes sociales ${ }^{1}$ du réseau des affaires sociales à Montréal en 1990-1991, difficultés appréhendées à partir d'incidents-critiques résultant de situations d'incompréhensions interculturelles survenues entre elles et leur clientèle-femmes et familles d'immigration récente.

Nous avions alors demandé aux intervenantes de sélectionner deux dossiers actifs de pratique sociale auprès des femmes immigrantes dans lesquelles elles avaient vécu des situations d'incompréhension interculturelle du fait que leurs valeurs, leurs normes étaient entrées en conflit avec celles de leur clientèle-familles et avaient produit un malentendu.

1. Le féminin est utilisé, les intervenantes sociales étant majoritairement des femmes. 
Outre ces situations abordées au cours d'entrevues semi-structurées de deux heures et demie chacune, l'ensemble de l'intervention sociale dans les dossiers était abordé, incluant les modalités de l'intervention interculturelle et féministe telles qu'elles sont comprises et pratiquées par elles.

Les personnes rencontrées ont été celles du réseau public des services sociaux de la région de Montréal, soit un échantillon d'intervenantes des Centres de services sociaux du Montréal métropolitain et de Ville-Marie (14) et des Centres locaux de services communautaires (26) parmi les plus " ethniques » de Montréal, c'est-à-dire ceux dont les territoires ont les plus hauts pourcentages de population d'origines ethniques diverses, tels qu'ils ont été déterminés par l'étude de Choinière (1990). Les supérieurs hiérarchiques ou les responsables des modules sociaux nous ont indiqué les intervenantes ayant le plus d'expérience auprès de la clientèle immigrante. Le traitement des données a été fait essentiellement par analyse de contenu.

Nous voudrions aborder, dans cet article, deux volets particuliers de cette recherche qui s'inscrivent en continuité de l'article de 1988, soit les problématiques sociales prioritaires des femmes immigrantes et l'intervention féministe auprès d'elles.

Au chapitre des problématiques sociales prioritaires affectant les femmes immigrantes, les écrits mettent l'accent principalement sur la remise en question des rôles sociaux qu'entraîne irrémédiablement la migration et sur les problèmes de santé physique et mentale.

\section{REMISE EN QUESTION DES RÔLES SOCIAUX}

Dans plusieurs communautés d'immigration récente, l'attribution des rôles hommes-femmes se fait souvent selon le mode patriarcal où l'homme est la figure dominante et détient une position privilégiée, les rôles étant spécifiques selon le sexe. L'homme est le pourvoyeur, considéré comme le chef de famille; les décisions importantes sont donc prises par lui, y compris, notamment, I'administration des punitions aux enfants (Hardy-Fanta, 1981). La femme y est avant tout mère et ménagère et le poids de la bonne marche du ménage et du bien-être de ses membres repose sur ses épaules. Rôles complémentaires différenciés, donc, et mondes séparés qui, sous l'effet de la migration, ont tendance dans un premier temps à se renforcer (Skodra, 1989).

Comme le soulignent Slucki (1979) et Landry (1990), I'homme sera porté à s'en tenir à des activités de type «instrumental » (orientées vers le présent et le futur) de contact avec l'environnement, alors que la femme s'en tiendra plutôt à des activités de type «affectif » (orientées vers le présent et le passé) de contact avec l'environnement antérieur. Cet état de faits a, bien sûr, des conséquences distingues en ce que l'homme établira ainsi des contacts 
qui faciliteront son adaptation autonome, alors que la femme contribuera à construire son isolement, considéré comme l'un des problèmes, sinon le plus important problème des femmes immigrantes.

La rupture avec le milieu d'origine, le temps variable de séparation des conjoints et le choc du nouveau contexte se conjuguent pour amener des remises en question dont l'issue est problématique. La confrontation avec la société d'accueil provoque une de ces remises en question, de même qu'une renégociation des rôles dans le couple.

La plupart des auteurs s'accordent à dire que l'insertion des femmes immigrantes sur le marché du travail est à la fois source de gains et de pertes. Sources de pertes, parce que leur insertion se fait souvent dans des secteurs d'emploi où les conditions de travail sont très difficiles. Source de gains aussi, toutefois, parce que, à travers le travail, la confiance en soi, la conscience d'améliorer sa situation et une plus grande participation aux décisions qui les concernent s'installent, sans oublier une sécurité financière accrue (Morokvasic, 1984; Lock et Dunk, 1987). Juteau (1991), dans sa recherche auprès des Salvadoriens de Montréal, émet l'hypothèse que séparation et divorce en tant qu'issues de la renégociation des rôles dans le couple sont reliés à l'intensité de la remise en question et à la façon qu'a chacun des partenaires de l'aborder. Elle souligne aussi que les femmes salvadoriennes au Québec se sentent plus reconnues, ont un meilleur accès au marché du travail, un plus grand contrôle de leur situation financière résultant de leur salaire ou de l'aide sociale de l'État, ce qui n'est pas sans créer des tensions dans le couple. Devant ces tensions, le couple soit resserrera ses liens dans un effort d'adaptation au nouveau contexte, soit se brisera parce que la rupture sera vue comme seule capable de résoudre le problème (Hartman et Hartman, 1983). Chez ce groupe, Juteau (1991) note un taux élevé de séparations et de divorces résultant des tensions provoquées par la migration, ce qui n'est pas toujours le cas, comme le fait remarquer Gavaki (1979) à propos des Grecs de Montréal, chez qui l'instabilité maritale et le divorce sont très faibles. Selon nous, cette différence entre Grecs et Salvadoriens s'explique par le type de migration: dans le premier cas, il s'agit de migrants, alors que dans le deuxième il s'agit de réfugiés. La migration des réfugiés, souvent involontaire et peu préparée, devient une situation de fragilisation qui contient un potentiel plus élevé de tensions pour le couple.

Dans d'autres cas, la confrontation avec une société plus égalitaire sur le plan des rapports hommes-femmes amène un renforcement des rôles traditionnels et un accroissement du pouvoir des hommes sur les femmes. Dans leur recherche auprès des femmes colombiennes, haïtiennes, grecques et portugaises, Labelle et al. (1987) ont constaté que le fait de gagner de I'argent ne changeait pas le rapport de pouvoir au sein du couple: I'homme y demeure l'autorité. Le travail salarié de la femme est vu comme un travail 
d'appoint, un supplément au budget familial, et celle-ci en retire peu de valorisation sociale. Les chercheures ajoutent que I'homme, qu'il soit conjoint, père ou frère continue d'exercer un certain contrôle sur les faits et gestes de la femme, plus particulièrement sur ses sorties, ses fréquentations et son comportement public.

Les exigences du travail salarié et la combinaison travail salarié - vie de famille, qui n'existait pas toujours ou qui se faisait plus facilement au pays d'origine, font que le couple doit vite affronter les tensions qui y sont inhérentes. Ainsi la question posée par Labelle et al. (1987 : 8) est on ne peut plus appropriée: "S'agit-il alors (pour les femmes) d'une accession à la modernité (par le transfert dans une société plus ouverte) ou d'un processus d'émancipation (la capacité pour la femme de gagner de l'argent remettant en cause son rôle subordonné) ou n'assiste-t-on pas plutôt au renforcement des rôles traditionnels et à l'accroissement du pouvoir des hommes du fait de la migration?» L'accroissement de ce pouvoir débouche parfois sur des situations de violence conjugale qui sont traitées de façon différente dans la société d'accueil. Souvent tempérées par des mécanismes d'intervention du milieu dans les sociétés d'origine, ces situations donnent lieu à des interventions des services sociaux publics auxquels les conjoints ne sont pas préparés.

Skodra (1989) en arrive aux mêmes conclusions que les auteures citées concernant les femmes immigrantes du Sud de l'Europe pour qui le travail salarié est une extension du travail ménager; elles travaillent en vue d'acheter à leurs familles les biens qu'elles fabriquaient et les services qu'elles fournissaient elles-mêmes auparavant et continuent de "prendre soin de leur famille», dans un esprit de service. Anthias (1983) va dans le même sens en affirmant qu'en dépit des effets positifs de la migration chez les femmes grecques en Angleterre, leur subordination à l'autorité mâle est toujours considérable.

\section{SANTÉ PHYSIQUE ET MENTALE}

Plusieurs écrits s'attardent à la santé des femmes immigrantes, à leurs préoccupations en ce domaine, à leurs expériences de contacts avec les services de santé et sociaux, à leurs attitudes à l'égard de ces services et de leurs agents.

Une constante se dégage des écrits recensés. Beaucoup de femmes immigrantes expriment leurs malaises et leurs insatisfactions à travers des somatisations et des symptômes divers qui sont autant d'appels à l'aide pour qui sait décoder les messages.

Lewin (1979) souligne la place qu'occupent la maladie et la consultation médicale dans l'univers de l'immigrante, plus particulièrement la 
femme latino-américaine émigrée sur la côte ouest américaine. Son article intitulé "La noblesse de la souffrance », rédigé à la suite d'une expérience $d^{\prime}$ intervention, nous informe que la maladie est en quelque sorte un "congé », pour la femme, de l'altruisme habituellement attendu d'elle, tout en attestant de son sacrifice passé. Sa maladie devient la preuve qu'elle s'est sacrifiée pour le bien de ses enfants et a été une mère vertueuse au point d'en devenir une «martyre ». La maladie lui accorde un répit des pressions de son rôle de mère « altruiste et oublieuse d'elle-même». La caution de la maladie l'autorise alors à s'occuper d'elle, à prendre congé des rôles domestiques et maternels et à se permettre ce loisir qu'est la visite au centre de soins et la rencontre d'autres femmes dans la même situation. Les femmes latino-américaines expriment aussi, à travers leur maladie, les insatisfactions reliées à leur situation de pauvreté et d'émigration.

Un phénomène semblable est approfondi par Wolf-Dresp (1985) au Massachusetts, à partir d'interviews avec des femmes portoricaines souffrant de nervios, trouble fréquemment mentionné en consultation par les femmes latino-américaines vivant aux États-Unis. Ce syndrome, causé par une perte, une maladie, des relations conflictuelles à l'intérieur de la famille ou avec la société en général, se manifeste par un état d'agitation et d'anxiété avec composantes somatiques, des changements d'humeur ou d'affect ou, encore, des changements d'habitudes alimentaires. Le terme nervios semble renvoyer à des émotions et à des conflits vécus d'une manière spécifique, propre à la communauté hispanophone. Il est frappant de constater, entre autres, que tous ces symptômes sont tournés vers la personne elle-même, qu'aucun n'exprime la déception ou la colère à l'endroit de causes extérieures pourtant souvent à l'origine des malaises.

Souffrant de nervios, la femme portoricaine souhaite obtenir soutien, aide et compréhension de sa famille ou de sa parenté. Ce soutien ne peut toutefois lui être apporté, en raison des changements socioculturels et politiques auxquels la famille a dû s'adapter et des nombreux facteurs de stress qui I'ont affectée. Plusieurs trouvent un soulagement dans des activités calmantes telles que l'activité physique et les techniques respiratoires. La consommation de thés médicinaux est aussi fréquente. Plusieurs, toutefois, consultent les professionnels de la santé, qui leur apportent surtout une aide médicamenteuse dont elles sont insatisfaites.

Les sentiments d'inquiétude, de solitude et surtout d'isolement, de dépression reviennent souvent chez les femmes immigrantes, lorsqu'on s'attarde à leur santé physique et mentale. Ainsi, à la suite de l'observation des contenus thématiques d'un groupe d'intervention auprès de Portoricaines déprimées, Comas-Diaz (1984) nous fait part de ses constatations. Elle explique que la femme portoricaine se conforme aux stéréotypes féminins en général et aux stéréotypes culturels en ce qui a trait à son rôle, faisant d'elle 
une personne dépendante, non affirmative, soumise, ce qui contribue à sa dépression. Par ailleurs, les facteurs qui affectent l'ensemble des Portoricains aux États-Unis, tels la pauvreté, les problèmes linguistiques et les barrières culturelles, influencent aussi sa santé générale. La rupture des routines culturelles, telles que les façons de s'alimenter, de se vêtir, de se comporter en société, et les attaques à leurs valeurs par la culture dominante font dire à l'auteure que l'identité de ces femmes est menacée et que cela affecte leur santé mentale.

Skodra (1989), dont la thèse de doctorat porte sur l'intégration des femmes immigrantes du Sud de l'Europe, explique que des symptômes somatiques viennent se greffer avant tout sur des situations de vie difficiles: conditions de travail salarié pénibles, cumul travail salarié - travail ménager, conditions de logement insatisfaisantes, habiletés de langage réduites, isolement. Ces manifestations somatiques comprennent maux de tête, difficultés respiratoires, insomnie, sentiment d'inutilité et de désespoir, le tout culminant en des malaises physiques reliés au fait de «trop penser et de trop s'en faire». Considérant leurs soucis comme étant du domaine privé et estimant qu'ils sont secondaires par rapport à ceux du mari, des enfants et de l'entourage, elles ne communiquent pas leurs préoccupations et les malaises physiques deviennent une réponse normale au stress qu'elles vivent. Lorsque, à la suggestion de la famille et des amis, elles consultent un médecin, des tranquillisants leur sont prescrits. Les malaises persistent, les femmes sont portées à prendre davantage de médicaments jusqu'à ce qu'elles soient dirigées, plus tard, vers un «spécialiste des nerfs » qui leur prescrit des antidépresseurs et quelquefois des antipsychotiques. Les femmes cessent alors de "s'en faire», mais se sentent fatiguées et endormies. Elles ne peuvent travailler à l'extérieur, non plus que faire leur travail domestique, ce dont les maris et l'entourage se plaignent. Cela ramène alors les femmes au traitement médical initial et aux malaises physiques, la boucle est bouclée.

Nous trouvons chez Lock et Dunk (1989) un écho aux malaises identifiés par Wolf-Dresp et Skodra sous le terme de nervios. Leur étude auprès d'une centaine de familles grecques de première génération à Montréal révèle la présence d'une maladie qu'on désigne sous le nom de nevra et qui s'apparente beaucoup à la nervios déjà mentionnée. Tout comme Skodra, Lock et Dunk affirment que ces malaises sont le reflet des problèmes divers que les familles rencontrent. Plus acceptables chez les hommes, ces malaises se manifestent différemment chez les femmes et sont le résultat de « circonstances exténuantes » La première cause associée au fait de souffrir « des nerfs » est le fait de ne pouvoir obtenir d'aide du conjoint ou des enfants à la suite de demandes répétées. Ainsi les femmes dont les maris s'en tiennent à des valeurs traditionnelles, contrôlant leurs sorties et leurs contacts avec l'extérieur, se sentant menacés dans leur philitomo (équivalant du machisme pour 
les Latinos-Américains), sont plus susceptibles de souffrir de nevra. La deuxième cause a trait aux soucis, reliés au travail salarié, à la double tâche, au logement, à la pauvreté de la famille, au climat et quelquefois à des conflits avec la parenté. La «maladie des nerfs » ou «nevra » chez les femmes exprime alors essentiellement des sentiments de frustration (quelquefois de colère), de manque de pouvoir ou de contrôle sur des situations immédiates de vie et d'anxiété devant les événements futurs.

Lorsque ces sentiments deviennent aigus, ils se transforment en maladie pour laquelle la femme consulte et reçoit habituellement une médication. Cette médication se révèle insuffisante si elle n'est pas accompagnée de modifications de comportement dans l'entourage et de changement des «circonstances accablantes».

\section{PROBlÉMATIQUES SOCIALES: DONNÉES DE LA RECHERCHE}

Au moment de la rencontre avec les intervenantes sociales du Réseau, nous avons relevé les problématiques sociales à l'intérieur desquelles les femmes immigrantes avaient été l'objet d'interventions sociales. Ainsi, nous posions, au début de l'entrevue, la question suivante: Pourquoi cette personne vous a-t-elle été envoyée? Dans les soixante dossiers discutés au cours des entrevues de recherche, 117 raisons de consultation ont été répertoriées.

Nous les avons classées en trois catégories, soit des problèmes reliés à l'enfance: 52 raisons, $45 \%$; des problèmes reliés aux femmes elles-mêmes: 37 raisons, $32 \%$; d'autres problèmes sociaux: 27 raisons, $23 \%$. Ce qui frappe d'abord, c'est la désignation des problèmes en rapport avec le mandat des services sociaux, avant tout centrés sur les enfants. Ainsi la majorité des interventions sont centrées sur la petite enfance (contraception, avortement, grossesse, accompagnement pré et postnatal) pour les CLSC et sur l'enfance (négligence et protection) pour les CSS. Des problématiques touchant directement les femmes viennent en second et totalisent $32 \%$ des raisons d'intervention; il s'agit alors de problématiques de violence conjugale (32\%), de santé mentale ( $27 \%$ ) (anxiété, angoisse, fragilité émotive, désorganisation, dépression, troubles émotionnels), d'isolement (22\%), de séparation/divorce $(16 \%)$. Enfin, d'autres problèmes sociaux ont été l'objet des interventions dans $23 \%$ des cas. Il s'agit de situations socio-économiques difficiles, de logement ou de travail inadéquat, de problèmes d'adaptation. Cette catégorie vient indéniablement compléter la deuxième car, comme nous l'avons vu dans la recension des écrits, plusieurs auteurs relient les problèmes de santé physique et mentale des femmes à leur situation de pauvreté, aux barrières linguistiques et culturelles, à leurs conditions de travail salarié et domestique, à leurs condition de logement, à leur isolement. 
Il demeure que 32 pour 100 seulement des motifs d'intervention ont trait à des problèmes reliés spécifiquement au vécu des femmes, ce qui pose le problème du manque de services sociaux publics accessibles aux femmes immigrantes. En raison du mandat des services sociaux publics, les femmes comme telles ne font pas l'objet d'interventions spécifiques. C'est donc par I'intermédiare de leurs enfants, qui eux font l'objet d'interventions prioritaires, qu'elles sont rejointes, à travers leur rôle de mère; leurs rôles sociaux sont nécessairement abordés par ce moyen. Quelques intervenantes ont mentionné l'aspect positif de cette situation dans le sens que les enfants permettent un accès aux femmes qui, peut-être, ne seraient pas autrement rejointes. Il est conséquemment nécessaire et souvent souhaitable, dans un premier temps, de se focaliser sur la famille, le couple et quelquefois le réseau.

\section{INTERVENTION FÉMINISTE}

Lorsque le sujet de l'intervention féministe auprès des femmes immigrantes était abordé, la question posée était la suivante: Connaissez-vous l'approche féministe? La définition suivante de l'approche était ensuite donnée: une approche qui situe les problèmes spécifiques vécus par les femmes dans l'ensemble de leur situation de subordination sociale, qui remet en question les rôles sociaux attribués aux femmes et les invite au changement personnel et social. Nous demandions alors aux intervenantes si, telle que définie, elles connaissaient l'approche et la pratiquaient. Nous avons alors constaté que 89 pour 100 des intervenantes connaissaient l'approche et que, telle qu'elle était définie, 75 pour 100 l'appliquaient dans leur intervention auprès des femmes immigrantes. Nous nous sommes alors intéressées aux modalités d'application de l'approche auprès de cette clientèle spécifique et avons obtenu divers types de réponses que nous voulons maintenant aborder en même temps que nous réfléchirons plus largement au sujet de l'intervention féministe auprès des femmes immigrantes.

Rappelons que l'intervention féministe - ou « counseling » féministe s'est développée à l'intérieur d'un questionnement sur la santé mentale des femmes au cours des années 60 et 70 (Corbeil et al., 1983; Valentich, 1986; Bourgon et Corbeil, 1990). Ce questionnement faisait suite à des études démontrant que les femmes consultaient davantage que les hommes, que des médicaments affectant le système nerveux central leur étaient prescrits plus souvent qu'aux hommes, qu'elles étaient vues par des thérapeutes souvent sexistes, c'est-à-dire ayant des visions différentes de la santé mentale selon que l'on est homme ou femme, et qu'elles étaient confinées dans des rôles sociaux restreints vus comme limitatifs et insatisfaisants. 
Ce questionnement sur la santé mentale des femmes s'est fait à I'intérieur d'une remise en question plus large de la situation des femmes dans la société contemporaine. Des analyses ont été effectuées dans l'ensemble des pays industrialisés et des actions entreprises sur plusieurs fronts affectant la vie des femmes; I'intervention féministe est l'une de ces actions. Essentiellement, les intervenantes s'interrogent sur la façon dont elles interviennent auprès des femmes à la lumière des analyses produites par le mouvement féministe des années 60 et 70, mouvement social parmi les plus importants du $20^{\mathrm{e}}$ siècle et de nouvelles modalités d'intervention sont élaborées. Ce sont ces modalités qu'on a qualifiées d'intervention féministe (Corbeil et al., 1983).

Les intervenantes sociales ont été partie prenante de ce questionnement. «Ces intervenantes questionnent le fait que les femmes soient les principales clientes des services sociaux et de santé et exigent des pratiques plus conformes à cette réalité, c'est-à-dire des pratiques permettant d'abord et avant tout aux femmes de se prendre en main» (Bourgon et Corbeil, 1990: 211). Pour ces intervenantes, en raison de la discrimination qui s'exerce à leur endroit dans la société, les femmes vivent une situation d'inégalité et d'absence de pouvoir sur plusieurs plans. Les actions à entreprendre vont dans le sens de revendications pour une situation plus égalitaire et le respect des droits des femmes. C'est ainsi que des actions ont été entreprises au Québec, surtout depuis les années 70, pour effectuer des réformes affectant la maind'œuvre, l'éducation, la sécurité sociale, les services de santé, de logement et de consommation générale, finalement les services sociaux.

Une étude récente (De Koninck et Savard, 1992) tente de faire le bilan de ce type d'intervention au Québec essentiellement dans le champ social et de la santé. Les auteures tentent de cerner l'essentiel des pratiques autour des variables analyse, objectifs, stratégies. L'analyse est celle des rapports sociaux de sexe où les situations personnelles des femmes sont replacées dans la situation globale faite aux femmes dans la société et où les dimensions sociales sont mises en évidence.

Les objectifs de l'intervention sont de favoriser une prise de conscience des dimensions sociales des problèmes vécus par les femmes et des stéréotypes sexistes qui les limitent à des rôles de service dans le privé autant que dans le public. L'intervention, qui se fait préférablement en groupe, vise à la fois un changement personnel et un changement social des dimensions qui affectent directement la vie des femmes. Ce changement personnel vise l'acquisition par la femme d'un pouvoir sur sa vie, d'une autonomie concrète et affective bâtie sur I'estime, I'affirmation de soi et la capacité de faire ses choix. Le changement social passe par la solidarité avec d'autres femmes, l'engagement communautaire et les actions collectives. Le schéma (page suivante) élaboré par Ann Pâquet-Deehy (1986) et auquel nous avons participé dans une version antérieure traduit bien, à notre avis, les paramètres de l'intervention féministe. 


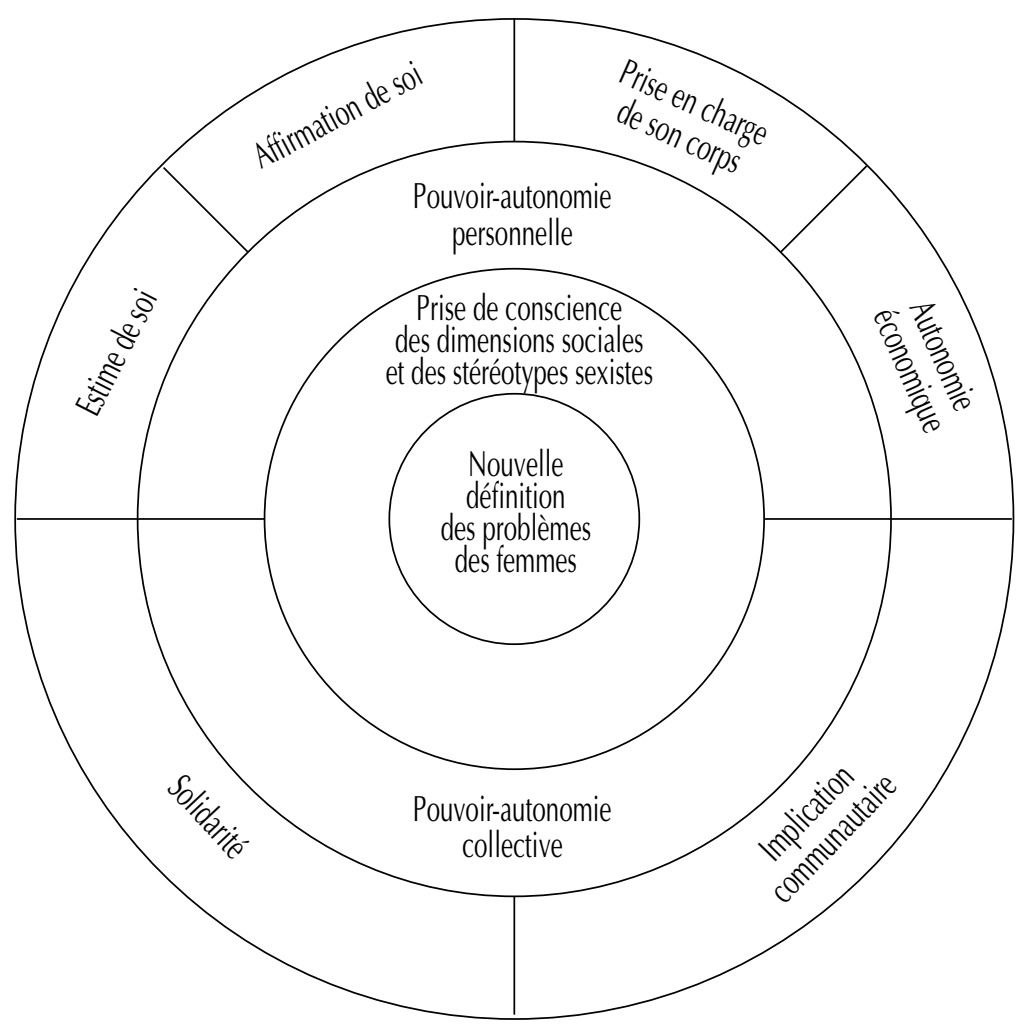

La stratégie privilégiée est celle d'un échange sur une base qui se veut la plus égalitaire possible. L'intervenante qui a effectué elle-même une démarche en relation avec sa situation de femme se perçoit comme membre de la catégorie sociale « femme » et cherche à aider d'autres femmes à partir de son expérience, d'un partage, d'un engagement véritable.

II semble donc que "les fondements théoriques de l'intervention féministe au Québec demeurent globalement tels qu'ils ont été énoncés au début des années 80 (Corbeil et al., 1983; Corbeil, 1979; Sturdivant, 1980; Guyon et al., 1980) et que ces intervenantes (dont les intervenantes sociales) s'y réfèrent à partir de quelques écrits» (De Koninck et Savard, 1992: 23). Certaines sont très critiques par rapport à la société actuelle et insistent sur I'intervention comme outil de contestation sociale, alors que d'autres l'assimilent à un ensemble de techniques non liées à une lecture féministe de la réalité et aux changements sociaux sur lesquels cette lecture débouche. Pour toutes, la socialisation des femmes et leur conditionnement à des stéréotypes sexistes sont parties intégrantes de l'analyse. Que faut-il en penser du point de vue de la pratique sociale auprès des femmes immigrantes? 


\section{INTERVENTION FÉMINISTE ET FEMMES IMMIGRANTES}

Nous dirions d'emblée que ces grands paramètres d'une intervention féministe auprès des femmes immigrantes sont les mêmes, soit l'analyse, les objectifs et les stratégies, chacune de ces dimensions tenant cependant compte des situations spécifiques des femmes immigrantes. Ainsi l'analyse des rapports sociaux de sexe inclura aussi celle des rapports sociaux de la société dont sont issues les femmes, rapports pouvant expliquer certaines de leurs difficultés actuelles. Skodra (1989: 185), critiquant les approches traditionnelles auprès des femmes immigrantes, souligne avec raison que: «An approach that focuses on immigrant women's counseling needs must recontextualize these women. That is, place them in their social, political and economic reality and do the same for the women's every day life experiences ». Lewis et Kissman (1989), relevant les points communs des approches féministe et interculturelle à partir de leur expérience de travail avec des femmes africaines-américaines, mentionnent que ces deux approches ont en commun cette compréhension des influences historiques, politiques et sociales sur la vie des personnes et leurs difficultés.

Nous reportant aux données de la recherche, mentionnons que les intervenantes sociales rencontrées renvoient à ce paramètre en disant qu'une intervention féministe réussie auprès des femmes immigrantes inclura " une évaluation de leur contexte général et global ». Cela apparaît particulièrement indiqué lorsqu'on se réfère aux problèmes sociaux des femmes immigrantes mentionnés dans les écrits et dans la recherche.

L'intervention féministe est applicable auprès des femmes immigrantes, mais elle doit être tempérée par d'autres facteurs qui affectent les femmes immigrantes et les immigrants en général; elle ne doit pas être proposée au premier plan.

La femme vit la subordination aussi à l'usine, par exemple vis-à-vis du contremaître. Si la syndicalisation est déjà là, que le harcèlement sexuel et racial est connu, dénoncé, c'est plus facile de faire prendre conscience à la femme d'un certain nombre de choix, de s'affirmer comme femme, de reconnaître qu'il y a situation d'oppression.

Les autres modalités d'une intervention féministe mentionnées sont:

1. l'évaluation des conceptions des rôles sociaux dans diverses cultures et leur degré de flexibilité;

2. la sensibilisation des communautés culturelles autres que québécoises à la conception des rôles sociaux de la société d'accueil;

3. I'acquisition de l'autonomie;

4. le respect du rythme de la cliente;

5. l'importance de ne pas nommer l'intervention féministe comme telle; 


\section{6. la création de groupes-soutiens;}

\section{I'orientation vers les ressources appropriées.}

Nous commenterons ces modalités en les insérant dans la discussion des variables de l'intervention féministe déjà amorcée.

Abordant les objectifs de l'intervention féministe, rappelons que, bien que les intervenantes se situent à différents pôles de la critique de la société quant à la situation faite aux femmes, toutes partagent minimalement cette analyse du processus de socialisation des filles et de leur conditionnement à des stéréotypes sexistes. En relation avec les femmes immigrantes, nous avons déjà abordé la remise en question des rôles sociaux et la renégociation de ces rôles dans le couple que provoquent la migration et la confrontation avec la société d'accueil. S'il est vrai que la situation de plusieurs femmes se trouve améliorée à la suite de la migration, pour d'autres ces gains ne sont qu'économiques, très tempérés sinon annulés par l'instabilité conjugale et familiale que ces mêmes gains engendrent (Hamer, 1981, dans Morokvasic, 1984).

On agira par conséquent avec prudence dans I'intervention auprès des femmes immigrantes à ce chapitre, évaluant d'abord, comme le mentionnent les intervenantes sociales qui ont participé à la recherche, la conception des rôles sociaux dans diverses cultures, leur degré de flexibilité et surtout le degré d'ouverture de la femme-cliente à la conception des rôles sociaux prônée par la société d'accueil.

Dans un de mes dossiers asiatiques, la mère était fâchée à l'endroit de sa fille, amoureuse d'un Canadien. Elle croyait qu'il revient aux parents de choisir le conjoint de leur enfant et que les jeunes ne doivent pas se fréquenter avant le mariage. Si j'avais été rapide et que j'avais dit que je croyais que les femmes sont les égales des hommes et qu'elles doivent choisir elles-mêmes leur conjoint j'aurais été mise à la porte de cette maison. C'est plus une question de culture ici que de rôles hommes-femmes. J'estime que je dois respecter les valeurs culturelles, ce qui ne veut pas dire que les gens sont satisfaits du déséquilibre des rôles hommes-femmes. Cette mère était d'ailleurs assez insatisfaite de son rôle et s'identifiait à sa fille dans son désir de plus de liberté. Elle remettait son rôle en question tout en défendant à sa fille certaines choses.

Il importe toutefois de créer, avant tout, comme le mentionnent les intervenantes, un climat de confiance et de sécurité qui puisse permettre à la femme immigrante de traverser la barrière qui sépare le monde privé du monde public dont fait partie l'intervenante. Skodra (1989), à cet effet, parle d'un intérêt réel à écouter les problèmes des femmes et à en comprendre la signification (sans imposer son propre schème de référence) comme étant la clé d'une ouverture des femmes sur la sphère privée. Ce partage du privé crée à son tour une alliance intervenante - cliente, essentielle et indispensable 
au processus d'aide. Elle insiste sur l'importance d'être consciente de la façon dont la femme vit son appartenance culturelle à travers une série de "positions de vie», investies de peu de pouvoir et souvent contradictoires entre elles. L'intervention aide alors à ouvrir progressivement pour la femme de nouvelles possibilités, l'aide à définir de plus en plus ses situations de vie et à acquérir plus de contrôle sur elles. C'est ce que Guindon (1990) du Centre des femmes de Montréal appelle la stratégie « des petits pas », dans le respect du rythme de la cliente. Cet aspect de l'intervention féministe auprès des femmes immigrantes est d'ailleurs la plus fréquemment mentionnée par les intervenantes que nous avons rencontrées, plusieurs spécifiant que la situation des femmes peut se trouver aggravée si le rythme de l'intervention est trop rapide. Plusieurs recommandent également d'être attentives aux signes $d^{\prime}$ 'essoufflement des femmes ou encore aux signes trompeurs d'une adoption trop rapide des modèles de la société d'accueil. C'est ce que Abadan-Umat (1977) qualifie de «pseudo-émancipation».

Je pense que c'est toujours à la personne de décider de l'aide qu'on peut lui offrir, de décider si elle la veut ou si elle ne la veut pas, on doit se maintenir ouverte... Je pense qu'il faut attendre aussi le moment où les gens sont prêts.

Si on réalise qu'une directivité féministe peut mettre la relation d'aide en question dans le sens d'amener une prise de conscience trop rapide, vous allez la perdre complètement. Si la femme devient anxieuse ou coupable à cause de cette intervention, elle est contreindiquée. Ce qui est mêlant avec les femmes latino-américaines, par exemple, c'est qu'elles peuvent avoir un discours très féministe au niveau des idées alors qu'au niveau concret, une fois chez elles, c'est autre chose.

II ne faut pas que je brusque la cliente là-dedans, il ne faut pas que mes valeurs à moi de femme occidentale viennent dire: bon, fais table rase de ça, tu changes de culture, tu adoptes mes comportements... Il faut que tu restes avec ce qu'elles ont comme repères culturels et que tu travailles avec ce qui, pour elles, reste acceptable ou reste acheminable en pensant que, peut-être, il y a beaucoup de choses qui sont encore plus oppressantes...

Dans le domaine de la santé physique et mentale, au moment où apparaissent anxiété, angoisse, somatisations diverses, insomnie, sentiments d'inutilité et d'impuissance, fragilité émotive, l'intervention sociale demande beaucoup de doigté pour saisir les messages cachés derrière ces symptômes de nervios et de nevra et aider les femmes à rendre explicites leurs insatisfactions. Comme le mentionne Wolf-Dresp (1985), les sentiments d'impuissance, de frustration et possiblement de colère deviennent alors connectés aux facteurs de stress et des solutions peuvent être envisagées. 
Toujours au chapitre des objectifs visés par l'intervention féministe, l'acquisition par la femme d'un pouvoir sur sa vie, d'une autonomie concrète et affective bâtie sur l'estime et l'affirmation de soi vient également au premier plan. Cet objectif demeure, indéniablement, en ce qui concerne les femmes immigrantes. Les intervenantes sociales placent l'acquisition de l'autonomie au deuxième rang des modalités de l'intervention féministe auprès des femmes immigrantes. Les mots clés qu'elles utilisent pour désigner cet objectif sont acquisition ou renforcement d'un pouvoir personnel sur sa vie, connaissance et affirmation de ses droits, image positive de soi, croyance en son potentiel, sens de sa propre valeur.

Disons que ma démarche est féministe, elle est de les rendre autonomes. C'est ce qu'on appelle leur redonner du pouvoir, mais ce $\mathrm{n}^{\prime}$ est pas toujours facile.

Je vais essayer de renforcer les femmes victimes de l'abus du conjoint, du propriétaire du logement, du patron ou du contremaître au travail.

Je ne recommande pas trop de précautions, les femmes immigrantes doivent survivre et le plus tôt elles le font, le meilleur c'est et pour elles et pour moi. On doit encourager les gens à participer à la société et à ne pas demeurer en périphérie.

C'est aussi l'avis de Guindon (1990), qui affirme que devenir autonome dans la société d'accueil est l'un des objectifs les plus importants visés par I'intervention féministe qui se pratique au Centre des femmes de Montréal. L'acquisition de cette autonomie est toutefois graduelle et tient compte des habiletés et des handicaps des femmes à leur arrivée. En agissant d'abord sur le plan individuel pour éviter le cantonnement des femmes dans la sphère affective et leur isolement, le Centre, tout comme les intervenantes rencontrées au moment de la recherche, favorisent aussi les démarches de groupes, groupes de socialisation et d'entraide ou groupes éducationnels-informationnels, groupes-soutien, en somme, qui contribuent à créer des solidarités, à tisser des réseaux et à augmenter les habiletés sociales des femmes.

L'engagement communautaire, les actions collectives et sociales sont finalement le dernier volet des objectifs visés par l'intervention féministe. Ce dernier volet est très important en ce qui a trait aux femmes immigrantes, car plusieurs dossiers requièrent ce type $d^{\prime}$ actions pour progresser. Il en est ainsi du statut des femmes qui sont souvent "parrainées », ce qui limite leur accès à nombre de programmes sociaux et leur autonomie en général, de leur non-maîtrise des langues du pays d'accueil, de leur accès limité au marché du travail et aux services de garde, etc., tous domaines où l'action collective doit compléter l'action individuelle et de groupe pour être efficace. On observe d'ailleurs sur le terrain actuellement (De Koninck et Savard, 1992: 22) des aménagements des types d'interventions, « fruits de l'expérience acquise 
et de la diversification tant des problématiques abordées que des clientèles rejointes».

Si l'on s'attarde maintenant quelque peu à la stratégie privilégiée en intervention féministe, soit l'échange intervenante-cliente le plus égalitaire possible, il faut souligner qu'elle a été quelque peu révisée pour mieux apprécier la distance qui sépare inévitablement les deux personnes à partir de variables telles que la classe sociale, la formation professionnelle, l'appartenance institutionnelle et, dans le cas qui nous occupe, l'expérience de la migration et le vécu de minoritaire marginalisée. Avec raison, Skodra (1989: 200) souligne que « although women therapists share with the women clients the experience of being women, they hold different positions of class, education, work setting and different positions of power in society ». Dans leur bilan de l'intervention féministe, De Koninck et Savard (1992) mentionnent que plusieurs intervenantes s'interrogent sur ces différences objectives qui séparent les femmes et qu'elles en tiennent compte dans leurs interventions, assurant un rôle de leadership et de soutien désiré par certaines femmes. Pour ces intervenantes, il ne s'agit pas d'un rejet de l'approche et de sa stratégie, mais d'une certaine maturité qui impose une adaptation de la pratique. II demeure toutefois que le partage d'expériences et l'engagement personnel de I'intervenante prôné par l'intervention féministe sied particulièrement bien à plusieurs femmes immigrantes qui recherchent chez l'intervenante sociale une amie, une sœur, une mère à qui elles pourraient se confier à la façon dont elles l'ont déjà fait dans leur milieu d'origine où la relation d'aide s'exerce d'abord sur le plan familial et communautaire informel.

Nous voulons finalement revenir sur un aspect mentionné par les intervenantes sociales qui ont participé à la recherche, soit l'importance de ne pas «nommer » I'intervention féministe comme telle. La raison invoquée est que ce vocable est souvent inconnu des femmes immigrantes ou encore qu'il leur fait peur dans le sens qu'il met l'accent sur la promotion et le bienêtre de la femme à un moment où se vivent pour elle et sa famille la remise en question et la renégociation des rôles sociaux. Les intervenantes affirment utiliser une approche féministe, mais de façon discrète, sans I'afficher ouvertement. Nous croyons que cet aspect de l'intervention féministe traduit une certaine ambivalence. De Koninck et Savard (1992) ont observé la même chose dans leurs entrevues au cours desquelles les intervenantes, disposant depuis quelques années d'une marge de manœuvre plus restreinte dans plusieurs milieux institutionnels, disent continuer de pratiquer l'intervention féministe sans la nommer. Ces intervenantes rattachent leur position au fait que l'intervention féministe, tributaire du mouvement des femmes, partage avec celui-ci depuis le début des années 90 un poids politique diminué. Le type d'argumentation n'est pas le même que dans le cas des femmes immigrantes, mais il traduit, croyons-nous, le même souci de ne pas faire peur, 
de ne pas choquer dans une période où le mouvement féministe se fait partout très discret. N'y a-t-il pas toutefois contradiction, comme De Koninck et Savard le soulignent, entre le fait de pratiquer une forme d'intervention tributaire d'un mouvement social qui vise des changements sociaux et le fait de ne pas la nommer explicitement?

\section{CONCLUSION}

L'intervention féministe a définitivement atteint une légitimation dans le champ social et de la santé. "Sa reconnaissance en tant qu'approche acceptable, acceptée et efficace » (De Koninck et Savard, 1992: 44) ne fait pas de doute, le bilan autant quantitatif que qualitatif est impressionnant. En effet, les problématiques couvertes, la clientèle rejointe, les lieux d'intervention et le nombre d'intervenantes concernées autant que ses aspects plus qualitatifs, tels les objectifs visés et les modalités d'intervention, amènent à conclure qu'il faut désormais compter l'intervention féministe au nombre des actions d'importance en ce qui a trait aux femmes et à une clientèle plus large.

Son évolution et son application à des catégories particulières de femmes, telles les femmes immigrantes, sont toutefois plus imprécises et doivent faire I'objet de réflexions plus poussées.

Le contexte évolutif de l'intervention féministe est tributaire du mouvement des femmes à l'intérieur duquel l'intervention s'est développée. Or, ce mouvement et le féminisme en général traversent une crise liée à celle de l'ensemble des mouvements sociaux en période de difficultés économiques et de retour à des valeurs conservatrices en certains domaines. C'est ce qui fait dire à certains que non seulement le féminisme n'est plus nécessaire, mais qu'il est dépassé.

Toutefois, rien n'est moins vrai, particulièrement en ce qui a trait aux femmes immigrantes pour qui une intervention appropriée à partir d'une compréhension juste de leurs problèmes reste à développer et à raffiner. Ainsi certaines interventions féministes pratiquées dans le champ de la périnatalité (pratique des sages-femmes, humanisation des soins de naissance, accompagnement), de la planification des naissances (Centre de santé pour femmes) et de la maternité (Corbeil et Descarries-Bélanger, 1987) pourraient être revues du point de vue des femmes immigrantes qui seraient d'autant plus facilement atteignables qu'elles valorisent souvent en priorité leur rôle de mère. 


\section{Références bibliographiques}

ABADAN-UNAT, N. (1977). «Implications of migration on emancipation and pseudoemancipation of Turkish women », International Migration Review, 11, Spring.

ANTHIAS, F. (1983). "Sexual divisions and ethnic adaptation: the case of Greek Cypriot women », dans A. Phizacklea (eds.), One way ticket, Migration and Female Labour. London: Routledge and Kegan Paul.

BOURGON, M. et C. CORBEIL (1990). «Dix ans d'intervention féministe au Québec: bilan et perspectives », Santé mentale au Québec, vol. XV, nº 1 .

CHOINIÈRE, R. (1990). La situation démographique et socio-économique de la population du Montréal métropolitain : principaux résultats du recensement de 1986 par CLSC et DSC. Montréal.

COMAS-DIAZ, L. (1984). "Content themes in group treatment with Puerto Rican woman », Social Work with Groups, vol. 7, n 3.

CORBEIL, C. et al. (1983). L'intervention féministe, l'alternative des femmes au sexisme en thérapie. Montréal : Saint-Martin.

CORBEIL, C. et F. DESCARRIES-BÉLANGER (1987). «La maternité: un défi pour les féministes », Revue internationale d'action communautaire, 18/58.

CORBEIL, J. (1979). "Les paramètres d'une théorie féministe de la psychothérapie», Santé mentale au Québec, "Vers une nouvelle pratique: La femme québécoise », V. 2.

DE KONINCK, M. et S. SAVARD (1992). De l'intervention féministe aux interventions féministes: des parcours multiples, une mémoire collective à construire. Rapport de recherche présenté au Conseil québécois de recherche sur la culture.

GAVAKI, E. (1979). "The Greek family in Canada: continuity and change and the process of adjustment », International Journal of Sociology of the Family, 9.

GUINDON, N. (1990). "Réflexion sur un type de pratique auprès des femmes immigrantes dans un centre de femmes », dans Actes du colloque Stratégies d'intervention auprès des femmes immigrantes: partager nos expériences et nos connaissances sur les diverses approches en travail social.

GUYON, L., R. SIMARD et L. NADEAU (1981). Va te faire soigner, t'es malade. Montréal: Stanké.

HARDY-FANTA, C. et E. MACMAHON-HERRERA, E. (1981). «Adaptating Family Therapy to the Hispanic Family», Social Casework, vol. 62, n 3.

HARTMAN, M.O. et H. HaRTMAN (1983). "The effect of immigration on women's roles in various countries », The International Journal of Sociology and Social Policy, vol. $3, \mathrm{n}^{\circ} 3$.

JUTEAU, F. (1991). "Divorce and the Migration Process among Salvadorians in Montreal », dans S.P. Shama, M. Ervin et D. Meintel (eds), Immigrants and Refugees in Canada. University of Saskatchewan, Regina.

Labelle, M., G. Turcotte, M. Kempeneers et D. Meintel (1987). Histoires d'immigrées. Montréal: Boréal.

LANDRY, M. et al. (1990). Dépaysées, au bout du monde. Montréal : Centre de ressources de la Troisième Avenue. 
LEGAULT, G. et M. LAFRENIÈRE (1992). «Situations d'incompréhensions interculturelles dans les services sociaux, problématique», Santé mentale au Québec, vol. XVII, $\mathrm{n}^{\circ} 2$.

LEGAULT, G. et E. ROJAS-BENAVENTE (1988). «Femmes immigrantes et travail social: une recension des écrits ", dans Service social, vol. 37, $\mathrm{n}^{\text {os }}$ 1-2.

LEWIN, E. (1979). "The nobility of suffering: illness and misfortune among Latin American immigrant women », Anthropology Quarterly, vol. 52, n 3.

LEWIS, E. et K. KISSMAN (1989). «Factors Linking Ethnic - Sensitive and Feminist Social Work Practice with African-American Women », ARETE, vol. 14, $\mathrm{n}^{\circ} 2$.

LOCK, M. et P. DUNK (1987). «My nerves are broken : the communication of suffering in a Greek-Canadian community », dans D. Coburn, C. D'Arcy, G. Torrance et P. New, Health and Canadian Society, Sociological Perspectives. Markham, Fitzhenry and Whiteside.

MOROCKVASIC, M. (1984). "Birds of Passage are also Women », International Migration Review, 18.

SKODRA, E. (1989). "Counselling immigrant women: feminist critique of traditional therapeutic approaches and evaluation of the role of the therapist», Counselling Psychology Quarterly, vol. 2, nº 2.

SLUZKI, C. (1979). "Migration and Family Conflict», Family Process, vol. 18, n 4. StURDivant, S. (1980). Les femmes et la psychothérapie. Bruxelles: Pierre Mardaga. VALENTICH, M. (1986). Feminism and Social Work Practice», dans F.J. Turner, Social Work Treatment, interlocking theoretical approaches, New York, Free Press.

WOLF-DRESP, C.S. (1985). «Nervios as a culture-bound syndrome among Puerto Rican women », Smith College Studies in Social Work, vol. 55, nº 2. 